\title{
Circular RNA expression and regulatory network prediction in posterior cingulate astrocytes in elderly subjects
}

\author{
Shobana Sekar ${ }^{1,2,3}$, Lori Cuyugan ${ }^{1,2}$, Jonathan Adkins ${ }^{1,2}$, Philipp Geiger ${ }^{1,2}$ and Winnie S. Liang ${ }^{1,2,3^{*}}$
}

\begin{abstract}
Background: Circular RNAs (circRNAs) are a novel class of endogenous, non-coding RNAs that form covalently closed continuous loops and that are both highly conserved and abundant in the mammalian brain. A role for circRNAs in sponging microRNAs (miRNAs) has been proposed, but the circRNA-miRNA-mRNA interaction networks in human brain cells have not been defined. Therefore, we identified circRNAs in RNA sequencing data previously generated from astrocytes microdissected from the posterior cingulate $(P C)$ of Alzheimer's disease (AD) patients $(N=10)$ and healthy elderly controls $(N=10)$ using four circRNA prediction algorithms - CIRI, CIRCexplorer, find_circ and KNIFE.

Results: Overall, utilizing these four tools, we identified a union of 4438 unique circRNAs across all samples, of which $70.3 \%$ were derived from exonic regions. Notably, the widely reported CDR1 as circRNA was detected in all samples across both groups by find_circ. Given the putative miRNA regulatory function of circRNAs, we identified potential miRNA targets of circRNAs, and further, delineated circRNA-miRNA-mRNA networks using in silico methods. Pathway analysis of the genes regulated by these miRNAs identified significantly enriched immune response pathways, which is consistent with the known function of astrocytes as immune sensors in the brain.

Conclusions: In this study, we performed circRNA detection on cell-specific transcriptomic data and identified potential circRNA-miRNA-mRNA regulatory networks in PC astrocytes. Given the known function of astrocytes in cerebral innate immunity and our identification of significantly enriched immune response pathways, the circRNAs we identified may be associated with such key functions. While we did not detect recurrent differentially expressed circRNAs in the context of healthy controls or AD, we report for the first time circRNAs and their potential regulatory impact in a cell-specific and region-specific manner in aged subjects. These predicted regulatory network and pathway analyses may help provide new insights into transcriptional regulation in the brain.
\end{abstract}

Keywords: Circular RNAs, Astrocytes, Posterior cingulate, Aging, Regulatory network

\section{Background}

CircRNAs are a class of endogenous, non-coding RNAs that form covalently closed continuous loops and that are pervasively expressed in eukaryotes [1-3]. Though RNA circularization events were reported in the 1970s and 1990s [4-6], they were disregarded as molecular artifacts arising from aberrant splicing. However, with the advent of next generation sequencing technology, coupled with the development of computational algorithms to specifically

\footnotetext{
* Correspondence: wliang@tgen.org

${ }^{1}$ Translational Genomics Research Institute, Phoenix 85004, AZ, USA

${ }^{2}$ Arizona Alzheimer's Consortium, Phoenix 85014, AZ, USA

Full list of author information is available at the end of the article
}

detect these back-splicing events, numerous circRNAs have been reported since 2012. CircRNAs exhibit cell type-, tissue- and developmental stage-specific expression [3, 7], and show evolutionary conservation between mouse and human $[2,3]$. Furthermore, circRNAs are highly abundant in the mammalian brain, compared to other tissues such as lungs, heart, kidney, testis and spleen in humans, as well as in mouse neuronal cell lines [8], and are derived preferentially from neural genes [9].

The abundance and evolutionary conservation of circRNAs suggests that they could play important roles in cellular processes. A few possible functions have been reported, including microRNA (miRNA) sponges [3, 6, $10,11]$, mediation of protein-protein interactions [12] 
and regulation of parental gene transcription [13]. Furthermore, a few circRNAs have been found to originate from disease-associated genomic loci, suggesting that circRNAs may regulate pathological processes [14-18]. Given these data, it is likely that circRNAs regulate RNA and protein networks, especially in the brain, but the regulatory pathways are still unknown.

In the present study, we characterized the expression and abundance of circRNAs in next generation RNA sequencing (RNAseq) data of human brain astrocytes. Astrocytes, the most abundant glial cells, play several essential roles in the central nervous system, including homeostasis [19], immunity [20] and energy storage and metabolism [21, 22]. We previously evaluated these astrocytes, which were derived from the posterior cingulate (PC) of Alzheimer's disease (AD) and healthy elderly control brains (age > 65), and identified ADassociated gene expression changes [23]. For this study, we used four circRNA prediction algorithms to identify circRNAs in these AD and control samples. Given the potential miRNA regulatory function of circRNAs, we then performed in silico identification of miRNA binding sites on the detected circRNAs, and further delineated putative circRNAmiRNA-mRNA networks in astrocytes. We describe here the first astrocyte-specific characterization of circRNAs and their interaction networks in elderly individuals.

\section{Results}

\section{CircRNA detection in PC astrocytes}

The RNAseq data generated from our previous study was used for analysis [23]. This data set was generated from 20 human PC astrocyte pools: 10 from late-onset $\mathrm{AD}$ (LOAD) brains and 10 from no disease (ND) healthy elderly control brains. Over $85,000,000$ reads were sequenced for each sample, with an average mapping percentage of 70.8. Using FASTQ files generated from sequencing, we ran four circRNA prediction algorithms - CIRCexplorer [24], CIRI [25], find_circ [3], and KNIFE [26], and detected a total of 4438 unique circRNAs with at least two supporting junction reads (Additional file 1: Table S1). Among the detected circRNA candidates, a total of 2331 circRNAs were identified in the AD samples and 2425 in the ND samples by at least one of the algorithms (Fig. 1a). While $80 \%$ of the detected circRNAs had less than ten supporting reads (Fig. 1b), 43 circRNAs had over 20 junction reads and were detected in more than one sample, and 31 circRNA candidates were detected in at least five samples with five or more supporting reads. Notably, the widely reported CDR1as circRNA was detected with a median read count of 52, by find_circ in all 20 samples and by CIRI in one of the samples. CircRNA 2:40,655,61240,657,444 (chromosome:start-end) was detected in 12 of the 20 samples by two, three or all four algorithms in each sample (Additional file 1: Table S1). Furthermore, 548 circRNAs detected in our dataset were also reported in the four studies deposited in circBase [27] (Additional file 1: Table S1); various cell lines and tissue types were evaluated in these studies, including cerebellum, diencephalon, SH-SY5Y cells, Hs68 cells, HeLa cells and HEK293 cells.

Among all identified circRNAs, 416 were on chromosome 1 (length $=249,250,621$ base pairs), while only eight were detected on chromosome Y (length $=59,373,566$ base pairs), consistent with previous findings that the number of circRNAs detected is proportional to the length of the chromosome [28]. Based on RefSeq annotations, we observed that $70.3 \%$ of our candidates were derived from exonic regions $(3,123 / 4,438)$, of which $94 \%$ $(2,936 / 3,123)$ were in coding DNA sequences (CDS; excludes untranslated regions) (Additional file 1: Table S1). Among the exonic circRNAs, $56.4 \%$ spanned one to 15 exons per circRNA, of which $20 \%$ were derived from single exons, while a small percentage of the exonic circRNAs (6.8\%) spanned over 100 exons per circRNA.

As previously reported [29], we observed that the overlap among the circRNAs detected by the different tools was low. Overall, 243 circRNAs were predicted by all four tools, while each tool also predicted unique circRNAs (KNIFE-1680, find_circ-1077, CIRI-488, CIRCexplorer-198; Fig. 1c). Most of the candidates called by all the tools originated from CDS $(242 / 243 ; 99.5 \%)$ as well as intronic regions $(232 / 243$; $95.5 \%)$, and $75 \%$ of the exonic candidates spanned two to six exons per circRNA. Further, the size distribution of all detected circRNAs, and the tool-wise and condition-wise distribution of the circRNAs, are summarized in Figs. 1d, e and f.

We next compared the relative abundance of circRNAs and corresponding linear RNAs using backspliced reads and linearly spliced reads with the same splice sites (Methods; Additional file 2: Table S2; Additional file 3: Figure S1). We observed that for 26 circRNAs, the circular-to-linear ratio was 10 or greater and the linear count was not 0 , such as circRNA 17: 48,823,196-48,824,063 from LUC7L3 (LUC7 like 3 premRNA splicing factor; average back-spliced reads: 413, average linear reads: 16.32 ) and 1:67,356,836-67,371,058 from WDR78 (WD repeat domain 78; average backspliced reads: 116.50 , average linear reads: 9.50). Further, $44.6 \%(1,983 / 4,438)$ had no expression of linear RNA and $45.5 \%(2,018 / 4,438)$ had higher expression of the linear RNA.

\section{MiRNA target prediction and delineation of circRNA- miRNA-mRNA regulatory networks}

Given the potential miRNA regulatory function of circRNAs, we next used the miRNA target prediction algorithms miRanda [30] and RNAHybrid [31] to predict the 
a

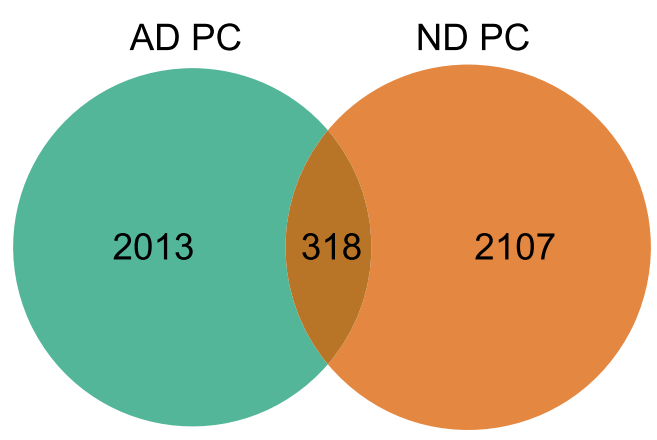

C

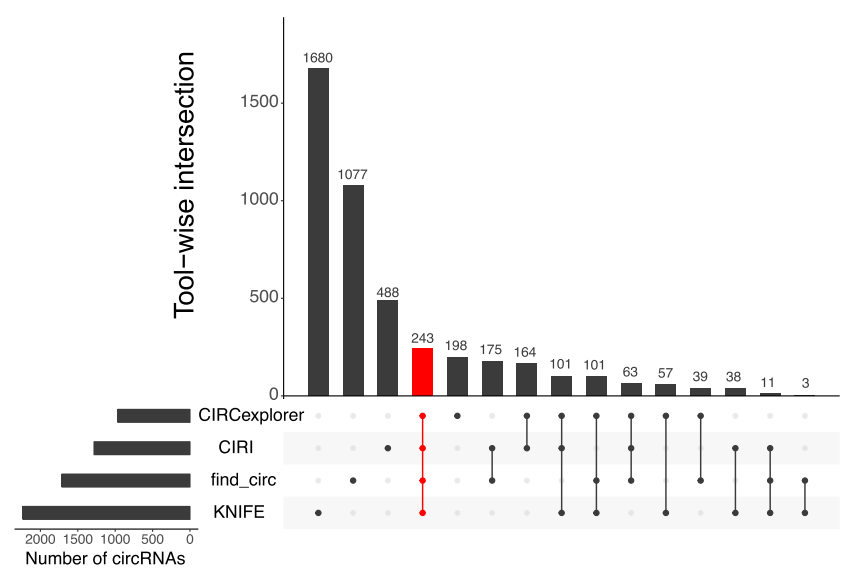

b

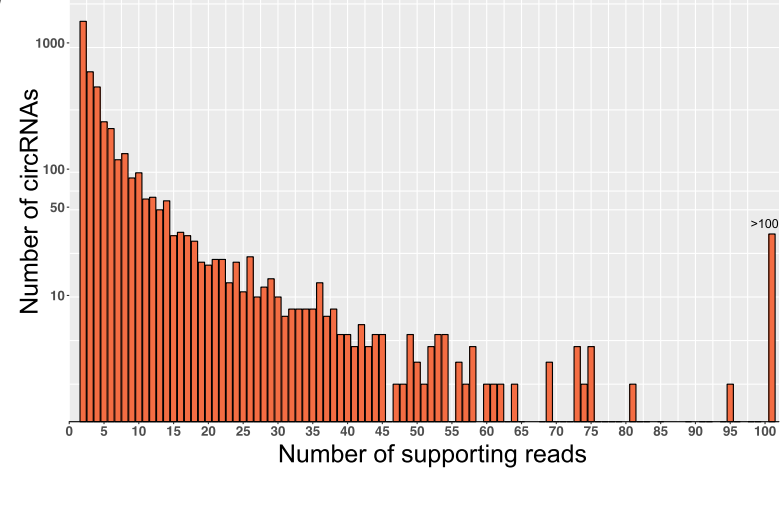

d

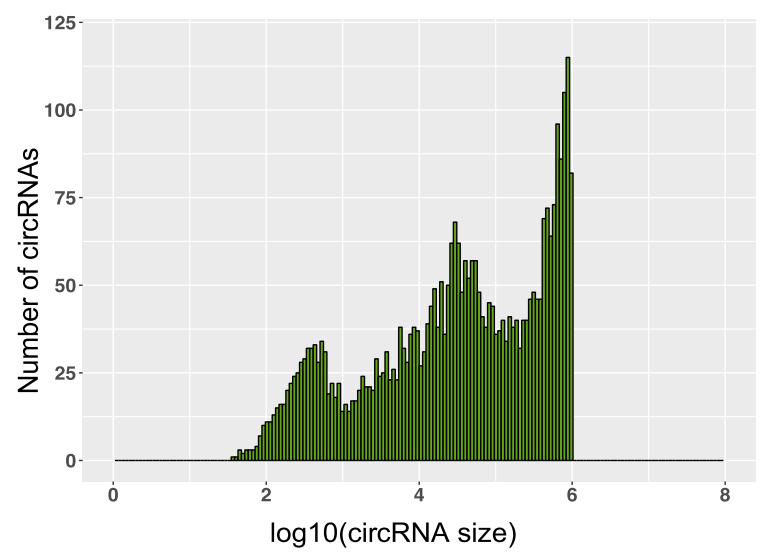

e

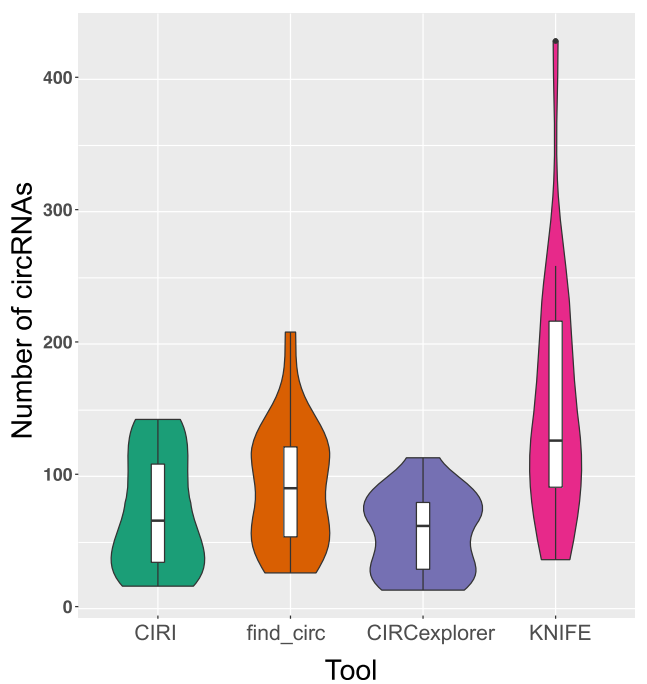

f

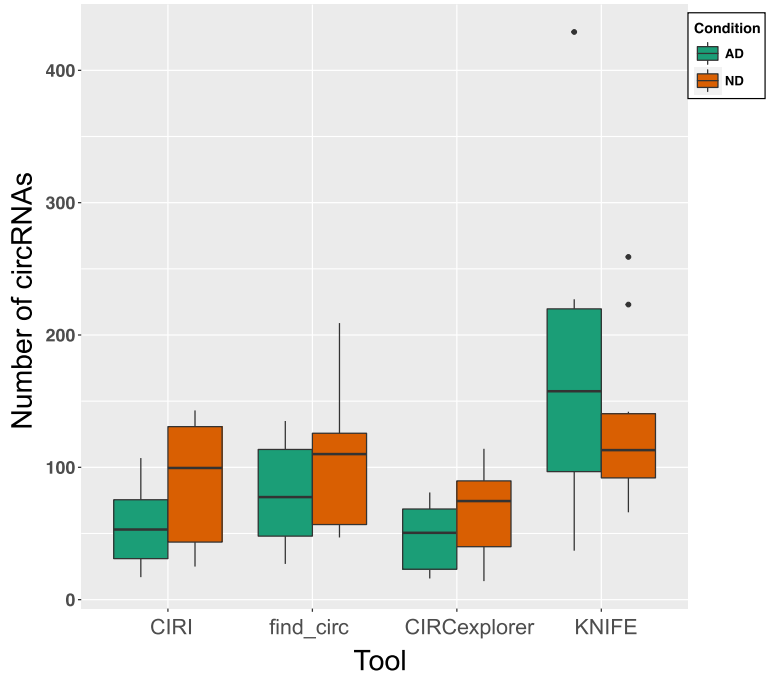

Fig. 1 Summary of circRNA prediction results. a Number of unique and common circRNAs in AD and ND PC. b Read count distribution of all detected circRNAs. $\mathbf{c}$ Intersection of circRNAs called by the four tools; the red bar indicates the number of circRNAs called by all four tools $\mathbf{d}$ Size distribution of all detected circRNAs. e Violin plots indicating the number of circRNAs predicted by each tool across PC samples along with the probability density. $\mathbf{f}$ Number of circRNAs predicted by each tool across PC samples, condition-wise. AD, Alzheimer's disease; ND, no disease; circRNA, circular RNA; PC, posterior cingulate; bp, base pairs 
miRNA targets of the circRNAs detected in ten or more samples by at least one of the circRNA prediction algorithms ( $N=10$ circRNA candidates). Using a list of 2588 published miRNAs from miRBase [32], we detected 14,296 unique interactions between circRNAs and miRNAs that were predicted by both miRNA target prediction algorithms and having a miRanda match score > $=150$. These interactions represent binding sites for miRNAs on each circRNA candidate, predicted based on complementarity in the miRNA seed region (nucleotide positions 2-7 in the miRNA 5 '-end). 2398 miRNAs in the reference set were predicted to have binding sites in our input list of circRNAs. Among these, a set of 612 circRNA-miRNA interaction pairs were predicted to contain over 100 putative interaction sites by the miRanda algorithm (Additional file 4: Table S3). These 612 circRNAmiRNA interactions were predicted for six unique circRNAs and 448 unique miRNAs. Using Cytoscape [33], we visualized the circRNA-miRNA interaction network for these 612 interactions, wherein the edges between circRNAs and its target miRNAs are weighted by the number of predicted interaction sites for the circRNA-miRNA pair (Fig. 2a). CDR1as was predicted to have binding sites for 74 distinct miRNAs and 63 binding sites for miR-7 (Fig. 2b). According to miRTarBase [34], miR-7 interacts with 578 target genes, some of which include SNCA (synuclein alpha), EIF4E (eukaryotic translation initiation factor 4E), KMT5A (lysine methyltransferase 5A), MAPKAP1 (mitogen-activated protein kinase associated protein 1), and $M K N K 1$ (MAP kinase interacting serine/threonine kinase 1).

We further employed the list of miRNA-mRNA target interactions common in both miRTarBase and TargetScan [35] databases, to determine the target genes of the above detected miRNAs. Overall, there were 2530 target genes for our input list of 2398 miRNAs, of which 255 were also differentially expressed between the AD and ND groups based on DESeq2 analysis [36] of the linear RNAs (uncorrected $P<0.05$, Additional file 5: Table S4). Using this information about miRNA target mRNAs, we delineated a putative lowstringency circRNA-miRNA-mRNA network consisting of ten circRNAs, 53 miRNAs and 255 genes (Additional file 6: Figure S2). Further, we used the same list of circRNAs detected in ten or more samples by at least one of the circRNA prediction algorithms, and increased the filtering stringency criteria to include circRNA-miRNA interactions with a miRanda match score $>=180$. We also restricted the candidate miRNAs to those with mRNA targets showing differential gene expression (uncorrected $P<0.05)$ with a $\log 2$ (fold change) $\geq 2$ or $\leq-2$ between the $\mathrm{AD}$ and ND groups. Using this strategy, we established a high-stringency circRNA-miRNA-mRNA interaction network with four circRNAs, 11 miRNAs and 49 genes (Fig. 3, Table 1). Our overall workflow is outlined in Additional file 7: Figure S3.

\section{Pathway analysis}

MetaCore pathway analysis on the 255 filtered differentially expressed target genes from the previous analysis revealed 112 perturbed pathways (corrected $P<0.01$; Table 2, Additional file 8: Table S5). 23 of these were immune response-related, such as the IL- 4 and IL- 6 signaling pathways. This identification of impacted immune response pathways is consistent with the known function of astrocytes as immune sensors in the brain and aligns with our previous RNAseq study, which showed that immune system response pathways are impacted in AD PC

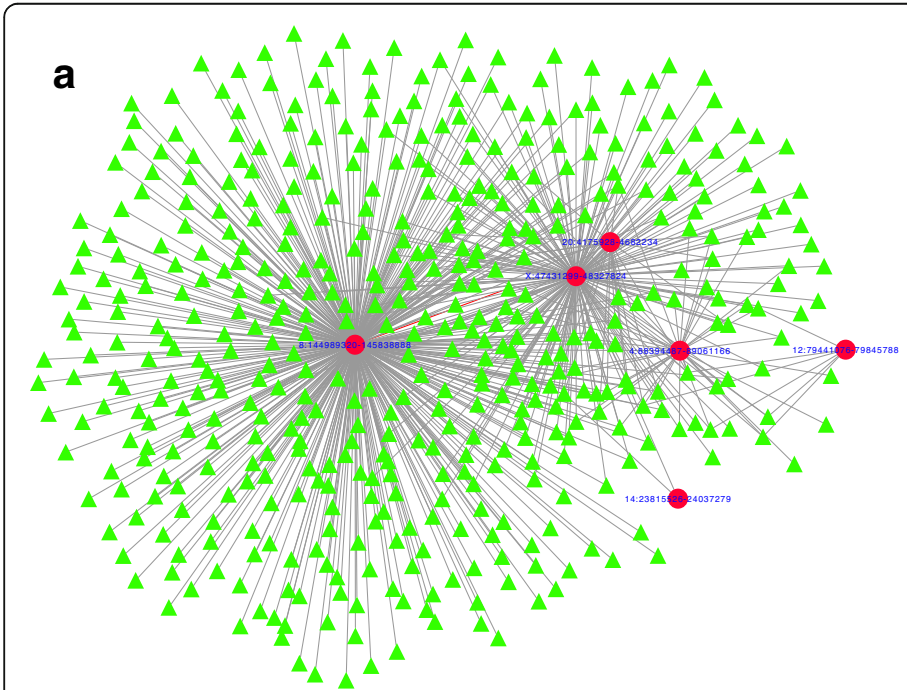

b

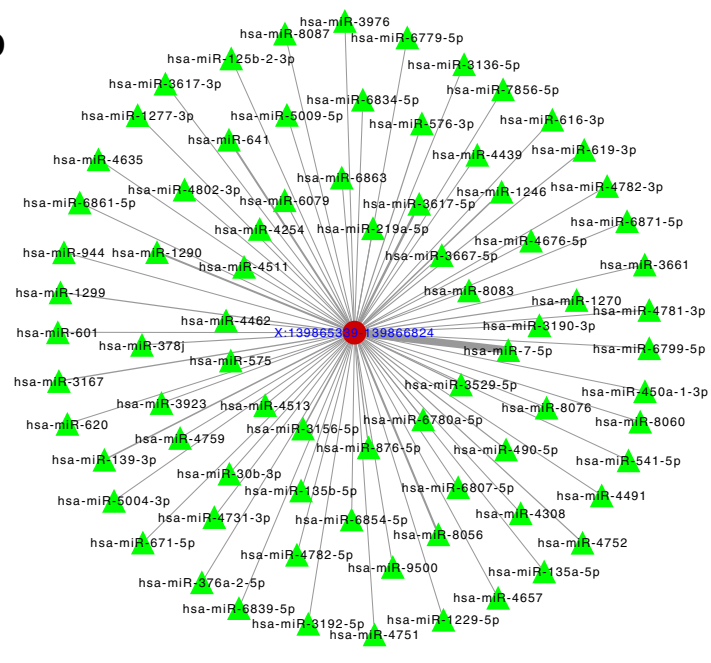

Fig. 2 circRNA-miRNA network. a circRNA-miRNA interactions with 100 or more predicted binding sites. Red circular nodes: circRNAs, green triangular nodes: miRNAs. $\mathbf{b}$ miRNA network of CDR1as. The edge thickness in $a$ and $b$ is weighted by the number of binding sites predicted for the circRNA-miRNA interaction. miRNA, microRNA 


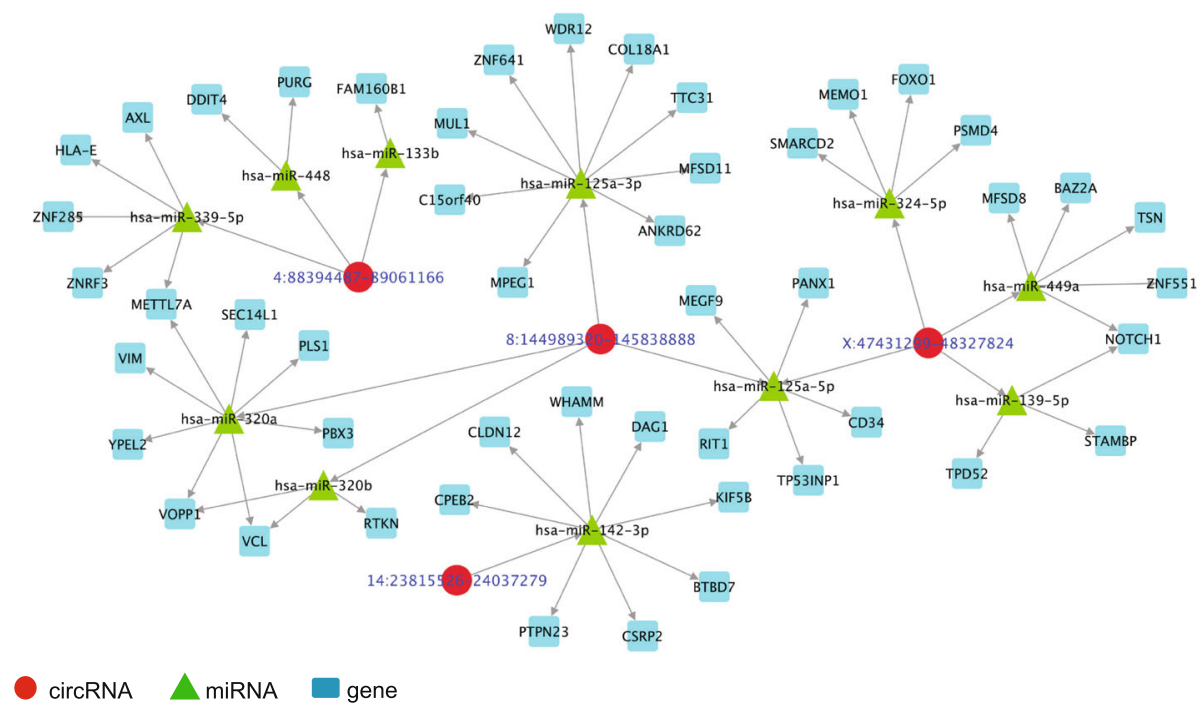

Fig. 3 High stringency circRNA-miRNA-mRNA regulatory network. Network of circRNA-miRNA-mRNA regulation for those circRNA-miRNA interactions predicted by both RNAHybrid and miRanda, with a miRanda match score $>=180$ and mRNA targets with differential expression (uncorrected $P<0.05$ ) and log2(fold change) $\geq 2$ or $\leq-2$. Red circular nodes: circRNAs, green triangular nodes: miRNAs, blue square nodes: genes. mRNA, messenger RNA

astrocytes compared to ND PC astrocytes [23]. Additionally, signal transduction pathways that may be perturbed include post-translational modifications (PTMs) in BAFFinduced signaling, mTORC2 downstream signaling and protein kinase A (PKA) signaling.

\section{Lack of circRNA differential expression in AD PC astrocytes}

We analyzed our catalog of circRNA candidates to determine whether there were circRNAs uniquely expressed in either the AD or ND cohort. Though there were over 2000 circRNAs unique to each group, we did not observe them to be recurrent in the samples within their respective group. The $\log 2$ (fold change) for all candidates calculated using DESeq2 are summarized in Additional file 1: Table S1. 93 circRNAs were unique to $\mathrm{AD}$ and called in at least two samples by at least one of the tools, and 82 circRNAs were unique to ND and called in at least two samples by at least one of the tools. These circRNA candidates were supported by at least two junction

Table 1 circRNA-miRNA-mRNA network elements for those circRNA-miRNA interactions predicted by both miRanda and RNAHybrid, with a miRanda match score $>=180$ and mRNA targets that are differentially expressed (uncorrected $P<0.05$ ) with log2(fold change) $>=2$ or $=<-2$ (high stringency network)

\begin{tabular}{|c|c|c|c|}
\hline Circular RNA & microRNA target & $\begin{array}{l}\text { Number of binding } \\
\text { sites predicted }\end{array}$ & Target genes (differentially expressed) \\
\hline$X: 47,431,299-48,327,824$ & hsa-miR-139-5p & 6 & NOTCH1, STAMBP, TPD52 \\
\hline $8: 144,989,320-145,838,888$ & hsa-miR-320a & 2 & METTL7A, PBX3, PLS1, SEC14L1, VCL, VIM, VOPP1, YPEL2 \\
\hline $8: 144,989,320-145,838,888$ & hsa-miR-320b & 2 & RTKN, VCL, VOPP1 \\
\hline$X: 47,431,299-48,327,824$ & hsa-miR-449a & 1 & BAZ2A, MFSD8, NOTCH1, TSN, ZNF551 \\
\hline $8: 144,989,320-145,838,888$ & hsa-miR-125a-3p & 1 & ANKRD62, C15orf40, COL18A1, MFSD11, MPEG1, MUL1, TTC31, WDR12, ZNF641 \\
\hline$X: 47,431,299-48,327,824$ & hsa-miR-125a-5p & 1 & CD34, MEGF9, PANX1, RIT1, TP53INP1 \\
\hline $8: 144,989,320-145,838,888$ & hsa-miR-125a-5p & 1 & CD34, MEGF9, PANX1, RIT1, TP53INP1 \\
\hline$X: 47,431,299-48,327,824$ & hsa-miR-324-5p & 1 & FOXO1, MEMO1, PSMD4, SMARCD2 \\
\hline $14: 23,815,526-24,037,279$ & hsa-miR-142-3p & 1 & BTBD7, CLDN12, CPEB2, CSRP2, DAG1, KIF5B, PTPN23, WHAMM \\
\hline $4: 88,394,487-89,061,166$ & hsa-miR-133b & 1 & FAM160B1 \\
\hline $4: 88,394,487-89,061,166$ & hsa-miR-448 & 1 & DDIT4, PURG \\
\hline $4: 88,394,487-89,061,166$ & hsa-miR-339-5p & 1 & AXL, HLA-E, METTL7A, ZNF285, ZNRF3 \\
\hline
\end{tabular}




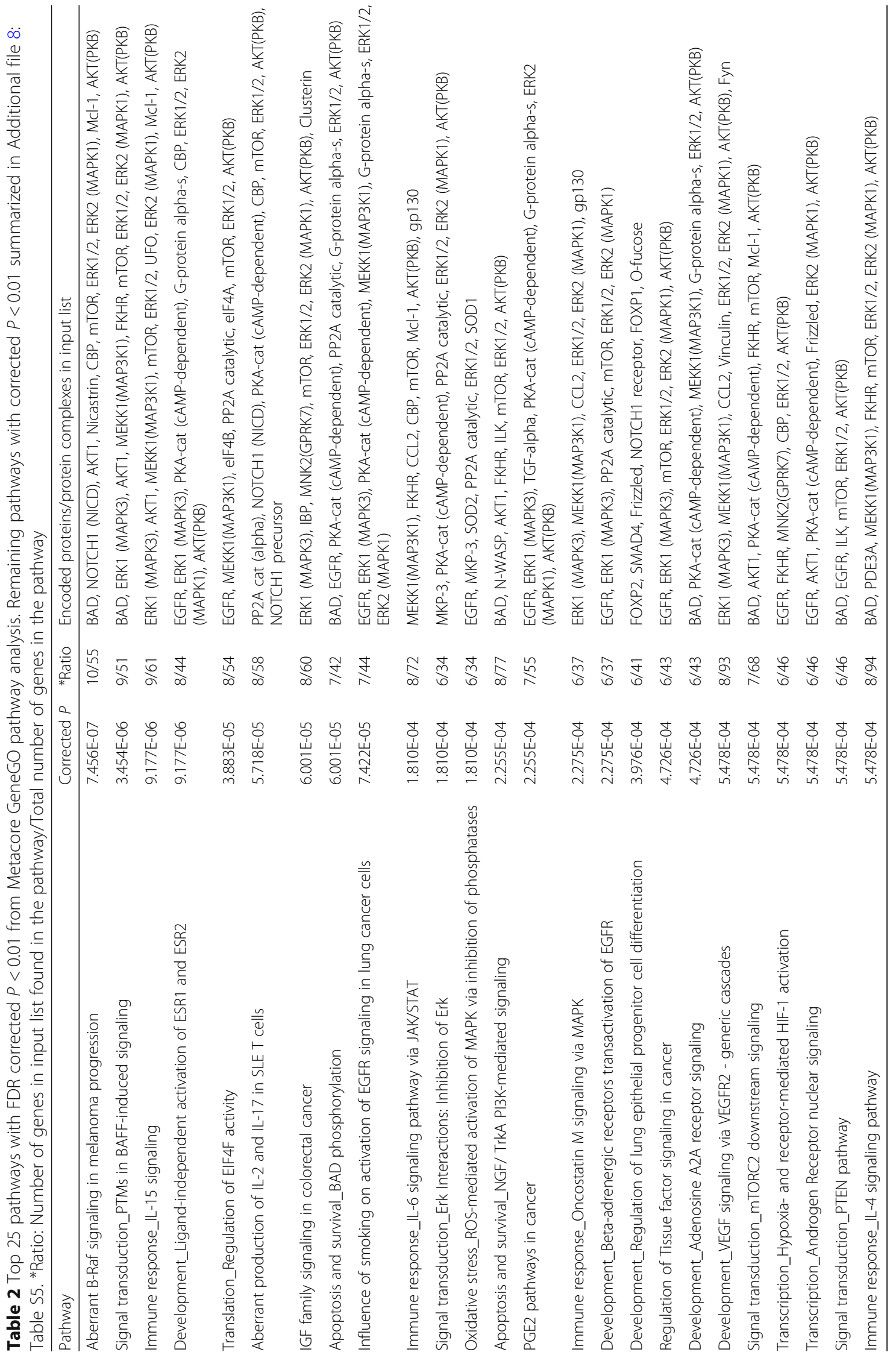


reads. To identify any differentially expressed candidates, we performed a Student's t-test on those circRNAs commonly called across the two groups. Only two circRNAs trending towards significance (uncorrected $P<0.05$ ) were identified and include 1:201,452,657-201,736,927 (uncorrected $P=0.015$ ) and $16: 1,583,657-2,204,141$ (uncorrected $P=0.046)$.

\section{Discussion}

CircRNAs, which are abundant in the mammalian brain, represent a recent addition to the class of noncoding RNAs. In this study, we detected astrocytic circRNAs using whole transcriptome RNAseq data obtained from the PC of $\mathrm{AD}$ and $\mathrm{ND}$ subjects, and outlined circRNA-miRNA-mRNA regulatory networks. Based on the results from four different circRNA detection algorithms, we identified over 4000 unique circRNAs across all samples, the majority of which were derived from coding exons. Although we did not identify circRNAs that were differentially expressed and recurrent in $\mathrm{AD}$ or $\mathrm{ND}$, we were able to delineate circRNA-miRNA-mRNA networks for the ten most recurrent circRNAs expressed across both groups, and also incorporate our previous differential expression analysis data from the linear mRNA. We observe that the majority of identified circRNAs are unique in the $\mathrm{AD}$ or ND groups and are not recurrent across the respective groups. This could be due to their low abundance in those samples, which may be below detection levels, or could be due to biological differences between the two groups, which requires further investigation. Pathway analysis on the differentially expressed miRNA target genes identified immune system related and signal transduction pathways. Notably, astrocytes are active players in cerebral innate immunity [37], and previous studies have reported that astrocytes respond to IL-4 signaling and potentially mediate between immune effector cells and nervous responders [38]. These predicted regulatory network and pathway analyses may help provide new insights into transcriptional regulation in the brain.

The circRNA CDR1as (also known as CiRS-7, a circRNA sponge for miR-7) was detected in all 20 of our samples and is a widely reported circRNA with 63 conserved seed matches for miR-7, indicating possible miR7 binding sites $[3,11]$. Interestingly, overexpression of CDR1as in zebrafish decreased the midbrain size, suggesting a functional role for CDR1as in the brain, while knockdown of CDR1as downregulated miR-7 targets in HEK293 cells [3]. This regulation is relevant since miR-7 plays a role in Parkinson's disease, stress handling and brain development [3, 39], and also has tumorsuppressive properties [39]. CDR1as also showed widespread expression in neuroblastoma and astrocytoma
[40]. However, expression of CDR1as was reduced in AD hippocampal samples about 0.18-fold compared to controls [15], which we did not observe in our PC astrocyte dataset. Apart from CDR1as, the tools also predicted circRNAs derived from genes including SLC8A1 (solute carrier family 8 - sodium/calcium exchanger member 1), which is under-expressed in hippocampal neurons from aged human brains [41], SYT1 (synaptotagmin 1), whose increase was correlated to age-related spatial cognitive impairment in mice [42], PSAP (prosaposin), which is increased in activated glia during normal aging in mouse brains [43], and FGF17 (fibroblast growth factor 17).

Although our dataset provides insights into the existence and abundance of astrocytic circRNAs in elderly individuals, there are a few limitations. Primarily, the whole transcriptome data we analyzed was not generated from samples that were depleted of linear RNAs using RNase R (ribonuclease R), an exoribonuclease that selectively digests linear RNA but leaves behind lariat or circRNA structures. Due to the presence of a larger pool of transcripts, which are mostly linear RNAs, RNAseq may not have comprehensively captured all the circRNAs in the samples. Notably, this enrichment step has been used by various groups to enrich for circRNAs for sequencing analyses [2, 3, 44].

Another limitation of bioinformatics-based circRNA detection is the highly divergent results produced by different algorithms. We observed this in our analyses and it has also been reported by two recent circRNA benchmark studies [29, 45]. The algorithms utilize different aligners, heuristics and filtering criteria, thus introducing 'blind spots' (false negatives) when addressing biases introduced by each method [46]. For example, find_circ and CIRI rely on filtering for GT-AG splice signals and thus may not capture candidates with non-canonical splice signals. Further, most tools use a read count filter, which may not be ideal for circRNAs with low expression relative to their linear host [47]. Given the low reliability on read counts, statistical approaches improve detection and classification of splice junctions, including novel ones [48]. Among the circRNA detection algorithms, KNIFE implements a logistic generalized linear model to distinguish true circRNAs, and is therefore able to identify circRNAs derived from non-canonical splice sites. Notably, KNIFE achieves a more balanced performance in terms of precision and sensitivity, compared to other circRNA detection algorithms, as described in one of the benchmarking studies [45]. We observed in our dataset that KNIFE detected more circRNAs compared to find_circ, CIRI and CIRCexplorer. Nonetheless, sequencing errors and technical artifacts introduced during RNAseq may still lead to false positive circRNAs, and hence statistical tests to estimate false discovery rates in circRNA detection need to be developed. 
While circRNAs have continued to gain attention as an abundant non-coding RNA species with potential regulatory functions, our understanding of their expression in various cell and tissue types remains limited. To address this challenge, we describe an analysis of astrocytic circRNAs in RNAseq data from elderly individuals, and we delineate potential circRNA-miRNA-mRNA regulatory networks. Given the role of astrocytes in signaling and synaptic modulation, and as immune sensors in the brain, the circRNAs we identified may be associated with such key functions. Further characterization using circRNA-enriched datasets will help us understand the atlas of circRNA expression in the context of specific cell types and conditions, including aging and $\mathrm{AD}$. In addition, downstream functional studies are needed to clarify how and whether circRNAs act as hubs for influencing protein expression and cellular processes. As we continue to piece together the factors involved in transcriptional regulation, we will both better understand basic cellular mechanisms and set the stage for developing improved therapeutic strategies for $\mathrm{AD}$ and other diseases.

\section{Conclusions}

In summary, we utilized astrocyte specific RNAseq data to identify astrocytic circRNAs in aged subjects $(N=20)$. Utilizing four circRNA prediction algorithms, we identified a total of 4438 unique circRNAs across samples, the majority of which were derived from exonic regions. The widely reported CDR1as circRNA was detected in all 20 samples with a median of 52 supporting reads. Given the putative miRNA regulatory function of circRNAs, we further performed in silico prediction of putative miRNA binding sites on the ten most recurrent circRNAs, and further delineated a low- and highstringency circRNA-miRNA-mRNA regulatory network. Pathway analysis on the genes from our low-stringency network revealed significantly impacted immune response pathways, which aligns with the known function of astrocytes as immune sensors in the brain. While we did not detect circRNAs recurrently expressed in the context of healthy controls or Alzheimer's, we are the first to report circRNAs and their potential regulatory impact in a cell-specific and region-specific manner in aged subjects. Continued analyses such as these sets the foundation for circRNA characterization and understanding their expression and regulatory networks in specific cell types and regions in the brain.

\section{Methods}

Sample acquisition, library preparation and paired-end sequencing

Detailed methods for sample acquisition, immunohistochemistry using an aldehyde dehydrogenase 1 family, member L1 (ALDH1L1) antibody, microdissection, RNAseq library preparation and sequencing of astrocytes are described in our previous publication [23]. Briefly, postmortem human brain samples were collected at the Banner Sun Health Research Institute's (BSHRI) Brain and Body Donation Program (BBDP) from 10 clinically classified LOAD subjects (4 males and 6 females; 5 $\mathrm{APOE} 83 / 4$ subjects and 5 APOE\&3/3 subjects) and 10 ND controls (6 males and 4 females; 5 APOE\&3/4 subjects and 5 APOE\&3/3 subjects). All subjects were enrolled in the BSHRI BBDP in Sun City, Arizona, and written informed consent for all aspects of the program, including tissue sharing, was obtained either from the subjects themselves prior to death or from their legally-appointed representative. The protocol and consent for the BBDP was approved by the Western Institutional Review Board (Puyallap, Washington). Clinical and pathological donor demographics are summarized in Additional file 9: Table S6. Approximately 300 astrocytes were laser capture microdissected from PC brain sections and total RNA was isolated from the cell lysates, followed by cDNA creation and library generation. Equimolar pools of libraries were sequenced by synthesis on the Illumina HiSeq2000 for paired 83 base pair reads.

\section{Data analysis}

The data analysis workflow is summarized in Additional file 7: Figure S3. Raw sequencing data, in the form of basecall files (BCLs), were converted to FASTQ format using Illumina's bcl2fastq conversion software and quality checked using FastQC [49]. To eliminate variance in circRNA detection that could arise due to differences in the number of sequencing reads, all FASTQ files were down-sampled to $85,547,262$ reads using seqtk [50]. The down-sampled FASTQ files were then run through four different circRNA prediction algorithms-CIRCexplorer (v1.1.10), CIRI (v2), find_circ (v1), and KNIFE (v1.4), using the parameter settings described in Additional file 10: Table S7. CircRNAs from each sample with at least two supporting reads were used for further downstream processing and analyses. CIRI produces 1-based circRNA coordinates, and was therefore converted to 0-based coordinates to be consistent with the other three algorithms. We then annotated our catalog of circRNA candidates using UCSC RefSeq annotations [51] and BEDtools [52].

The ratio of circular-to-linear RNA isoforms was calculated using the approach described in [8]. For each circRNA candidate, we used the number of back-spliced reads for circRNA quantification $\left(N_{c}\right)$ and the number of linear reads supporting the same $5^{\prime}$ or $3^{\prime}$ splice junction $\left(N_{l 5}\right.$ or $\left.N_{l 3}\right)$ as the number of linear RNA reads. The linear junction supporting reads were obtained by aligning 
our RNAseq data to the reference genome (GRCh37) using STAR [53].

$$
\text { Circular to linear ratio }=N_{c} / \max \left(N_{l 5}, N_{l 3}\right)
$$

\section{miRNA target prediction}

For circRNAs detected in at least $50 \%$ of the samples, we next conducted miRNA binding site prediction using the miRanda [30] and RNAHybrid [31] algorithms. The miRanda algorithm finds potential target sites for miRNAs in a genomic sequence using a two-step strategy. First, a dynamic programming local alignment is implemented between the miRNA sequence and the sequence of interest (circRNA sequence in this study), scoring the alignment based on sequence complementarity (match score). In the second step, the thermodynamic stability of the resulting RNA duplex is estimated based on the high-scoring alignments from the first phase. The RNAHybrid algorithm finds the energetically most favorable hybridizations of a small RNA to a large RNA. Only those circRNA-miRNA interactions predicted by both the algorithms are used for our downstream network construction and analyses. From the list of commonly predicted circRNAmiRNA interactions, we filtered for those having a miRanda match score $>=150$.

\section{circRNA-miRNA-mRNA network construction}

miRNA-mRNA interactions that are common in both miRTarBase [34] and TargetScan [35] were then used to determine the gene targets of each filtered miRNA and compared with genes identified using differential expression analysis of the linear RNAs (uncorrected $P<0.05$; DESeq2 performed as described in our previous publication). Using these data, we outlined a low-stringency circRNA-miRNA-mRNA regulatory network using custom python scripts and visualized the network using cytoscape. We further filtered for circRNA-miRNA interactions with miRanda match scores $>=180$ and miRNAs with mRNA targets showing differential expression (uncorrected $P<0.05, \log 2$ [fold change] $\geq 2$ or $\leq-2$ ) to outline a high-stringency circRNA-miRNA-mRNA network.

\section{Pathway analysis}

On the list of filtered miRNA target genes with DESeq2 uncorrected $P<0.05$, we performed pathway analysis using MetaCore GeneGO (v6.32.69020) from Thompson Reuters to predict pathways that are commonly impacted in the AD and ND groups. The results were filtered for enriched pathways with a false discovery rate (FDR)-corrected $P<0.01$.

\section{Additional files}

Additional file 1: Table S1. Master summary of all detected circRNAs (XLSX 559 kb)

Additional file 2: Table S2. Circular-to-linear ratios for all detected circRNAs (XLSX $342 \mathrm{~kb}$ )

Additional file 3: Figure S1. Circular-to-linear ratios. Ratio of average back-spliced reads to average linearly spliced reads for all detected circRNAs. (PDF $1075 \mathrm{~kb}$ )

Additional file 4: Table S3. CircRNA-miRNA interactions with $\geq 100$ predicted binding sites. (XLSX $48 \mathrm{~kb}$ )

Additional file 5: Table S4 DESeq2 analysis results for genes with uncorrected $P<0.05$, between AD and controls. (XLSX $229 \mathrm{~kb}$ )

Additional file 6: Figure S2. Low stringency circRNA-miRNA-mRNA regulatory network. Network of circRNA-miRNA-mRNA regulation for those circRNA-miRNA interactions predicted by both RNAHybrid and miRanda, with miRanda match scores $>=150$ and mRNA targets with differential expression (uncorrected $P<0.05$ ). Red circular nodes: circRNAs, green triangular nodes: miRNAs, blue square nodes: genes. (PDF $771 \mathrm{~kb}$ )

Additional file 7: Figure S3. Computational workflow outline and filtering criterion. PC, posterior cingulate; RNAseq, RNA sequencing; circRNA, circular RNA; miRNA, microRNA; mRNA, messenger RNA. (PDF 928 kb)

Additional file 8: Table S5. Pathways with corrected $P<0.01$, apart from the ones summarized in Table 2. (XLSX $14 \mathrm{~kb}$ )

Additional file 9 Table S6. Donor demographics. (XLSX 41 kb)

Additional file 10: Table S7. Tool parameters used for circRNA detection in this study. (XLSX $45 \mathrm{~kb}$ )

\section{Abbreviations}

AD: Alzheimer's disease; ALDH1L1: aldehyde dehydrogenase 1 family, member L1; BBDP: Brain and Body Donation Program; BSHRI: Banner Sun Health Research Institute; CDS: coding DNA sequences; CircRNA: circular RNA; FDR: False discovery rate; LOAD: Late-onset Alzheimer's disease; miRNA: microRNA; mRNA: messenger RNA; PC: posterior cingulate; RNAseq: RNA sequencing

\section{Acknowledgements}

We are grateful to the Banner Sun Health Research Institute Brain and Body Donation Program of Sun City, Arizona for the provision of human brain tissues for our previous study. The BBDP has been supported by the National Institute of Neurological Disorders and Stroke (U24 NS072026 National Brain and Tissue Resource for Parkinson's Disease and Related Disorders), the National Institute on Aging (P30AG19610 Arizona Alzheimer's Disease Core Center), the Arizona Department of Health Services (contract 211002, Arizona Alzheimer's Research Center), the Arizona Biomedical Research Commission (contracts 4001, 0011, 05-901 and 1001 to the Arizona Parkinson's Disease Consortium) and the Michael J. Fox Foundation for Parkinson's Research [54]. We would also like to thank TGen's Dr. Kendall Jensen and Dr. Elizabeth Hutchins for input and guidance, and Cynthia Lechuga for administrative support. Nancy Linford, PhD, provided editorial suggestions.

\section{Funding}

Research reported in this publication was supported by the National Institute on Aging (NIA) of the National Institutes of Health under award number P30AG019610, and the Arizona Department of Health Services award number ADHS14-052688. The content is solely the responsibility of the authors and does not necessarily represent the official views of the National Institutes of Health. The funders had no role in the study design, data collection and analysis, decision to publish, or preparation of the manuscript.

Availability of data and materials

All the RNAseq data generated in this study are accessible through the National Center for Biotechnology Information (NCBI) database of Genotypes and Phenotypes (dbGaP; accession\# phs000745.v1.p1), and 
data supporting our findings are included within the manuscript and additional figures/tables.

\section{Authors' contributions}

WL conceived the study. SS and WL performed all data analysis and interpretation and wrote the manuscript. LC, JA and PG made substantial contribution to data acquisition, performed library preparation and sequencing of all samples and also contributed to data interpretation. All authors read and approved the final manuscript.

\section{Ethics approval and consent to participate}

All subjects were enrolled in the BSHRI BBDP in Sun City, Arizona, and written informed consent for all aspects of the program, including tissue sharing, was obtained either from the subjects themselves prior to death or from their legally-appointed representative. The protocol and consent for the BBDP was approved by the Western Institutional Review Board (Puyallap, Washington).

\section{Competing interests}

The authors declare that they have no competing interests.

\section{Publisher's Note}

Springer Nature remains neutral with regard to jurisdictional claims in published maps and institutional affiliations.

\section{Author details}

${ }^{1}$ Translational Genomics Research Institute, Phoenix 85004, AZ, USA. ${ }^{2}$ Arizona Alzheimer's Consortium, Phoenix 85014, AZ, USA. ${ }^{3}$ Arizona State University, Tempe, AZ 85287, USA.

\section{Received: 19 December 2017 Accepted: 13 April 2018}

Published online: 09 May 2018

\section{References}

1. Salzman J, Gawad C, Wang PL, Lacayo N, Brown PO. Circular RNAs are the predominant transcript isoform from hundreds of human genes in diverse cell types. PLoS One. 2012;7(2):e30733.

2. Jeck WR, Sorrentino JA, Wang K, Slevin MK, Burd CE, Liu J, Marzluff WF Sharpless NE. Circular RNAs are abundant, conserved, and associated with ALU repeats. RNA (New York, NY). 2013;19(2):141-57.

3. Memczak S, Jens M, Elefsinioti A, Torti F, Krueger J, Rybak A, Maier L, Mackowiak SD, Gregersen LH, Munschauer M. Circular RNAs are a large class of animal RNAs with regulatory potency. Nature. 2013;495(7441):333-8.

4. Sanger HL, Klotz G, Riesner D, Gross HJ, Kleinschmidt AK. Viroids are singlestranded covalently closed circular RNA molecules existing as highly basepaired rod-like structures. Proc Natl Acad Sci U S A. 1976;73(11):3852-6.

5. Nigro JM, Cho KR, Fearon ER, Kern SE, Ruppert JM, Oliner JD, Kinzler KW, Vogelstein B. Scrambled exons. Cell. 1991;64(3):607-13.

6. Capel B, Swain A, Nicolis S, Hacker A, Walter M, Koopman P, Goodfellow P, Lovell-Badge R. Circular transcripts of the testis-determining gene Sry in adult mouse testis. Cell. 1993:73(5):1019-30.

7. Salzman J, Chen RE, Olsen MN, Wang PL, Brown PO. Cell-type specific features of circular RNA expression. PLoS Genet. 2013:9(9):e1003777.

8. Rybak-Wolf A, Stottmeister C, Glažar P, Jens M, Pino N, Giusti S, Hanan M, Behm M, Bartok O, Ashwal-Fluss R. Circular RNAs in the mammalian brain are highly abundant, conserved, and dynamically expressed. Mol Cell. 2015; 58(5):870-85.

9. You X, Vlatkovic I, Babic A, Will T, Epstein I, Tushev G, Akbalik G, Wang M, Glock C, Quedenau C. Neural circular RNAs are derived from synaptic genes and regulated by development and plasticity. Nat Neurosci. 2015;18(4):603.

10. Hansen TB, Wiklund ED, Bramsen JB, Villadsen SB, Statham AL, Clark SJ, Kjems J. miRNA-dependent gene silencing involving Ago2-mediated cleavage of a circular antisense RNA. EMBO J. 2011;30(21):4414-22.

11. Hansen TB, Jensen TI, Clausen BH, Bramsen JB, Finsen B, Damgaard CK, Kjems J. Natural RNA circles function as efficient microRNA sponges. Nature. 2013:495(7441):384-8.

12. Du WW, Yang W, Liu E, Yang Z, Dhaliwal P, Yang BB. Foxo3 circular RNA retards cell cycle progression via forming ternary complexes with p21 and CDK2. Nucleic Acids Res. 2016;44(6):2846-58.
13. Li Z, Huang C, Bao C, Chen L, Lin M, Wang X, Zhong G, Yu B, Hu W, Dai L. Exon-intron circular RNAs regulate transcription in the nucleus. Nat Struct Mol Biol. 2015;22(3):256-64.

14. Burd CE, Jeck WR, Liu Y, Sanoff HK, Wang Z, Sharpless NE. Expression of linear and novel circular forms of an INK4/ARF-associated non-coding RNA correlates with atherosclerosis risk. PLoS Genet. 2010;6(12):e1001233.

15. Lukiw W. Circular RNA (circRNA) in Alzheimer's disease (AD). Front Genet. 2013;4:307.

16. Bachmayr-Heyda A, Reiner AT, Auer K, Sukhbaatar N, Aust S, BachleitnerHofmann T, Mesteri I, Grunt TW, Zeillinger R, Pils D. Correlation of circular RNA abundance with proliferation-exemplified with colorectal and ovarian cancer, idiopathic lung fibrosis, and normal human tissues. Sci Rep. 2015;5:8057.

17. Li F, Zhang L, Li W, Deng J, Zheng J, An M, Lu J, Zhou Y. Circular RNA ITCH has inhibitory effect on ESCC by suppressing the Wnt/beta-catenin pathway. Oncotarget. 2015;6(8):6001-13.

18. Li P, Chen S, Chen H, Mo X, Li T, Shao Y, Xiao B, Guo J. Using circular RNA as a novel type of biomarker in the screening of gastric cancer. Clin Chim Acta. 2015:444:132-6.

19. Parpura V, Verkhratsky A. Homeostatic function of astrocytes: ca(2+) and $\mathrm{Na}(+)$ signalling. Transl Neurosci. 2012:3(4):334-44.

20. Jensen CJ, Massie A, De Keyser J. Immune players in the CNS: the astrocyte. Neuroimmune Pharmacol. 2013:8(4):824-39.

21. Tsacopoulos M, Magistretti PJ. Metabolic coupling between glia and neurons. J Neurosci. 1996;16(3):877-85.

22. Pellerin L, Magistretti PJ. Glutamate uptake into astrocytes stimulates aerobic glycolysis: a mechanism coupling neuronal activity to glucose utilization. Proc Natl Acad Sci. 1994:91(22):10625-9.

23. Sekar S, McDonald J, Cuyugan L, Aldrich J, Kurdoglu A, Adkins J, Serrano G, Beach TG, Craig DW, Valla J, et al. Alzheimer's disease is associated with altered expression of genes involved in immune response and mitochondrial processes in astrocytes. Neurobiol Aging. 2015;36(2):583-91

24. Zhang XO, Wang HB, Zhang Y, Lu X, Chen LL, Yang L. Complementary sequence-mediated exon circularization. Cell. 2014;159(1):134-47.

25. Gao Y, Wang J, Zhao F. CIRI: an efficient and unbiased algorithm for de novo circular RNA identification. Genome Biol. 2015;16:4-014. 0571-0573

26. Szabo L, Morey R, Palpant NJ, Wang PL, Afari N, Jiang C, Parast MM, Murry CE, Laurent LC, Salzman J. Statistically based splicing detection reveals neural enrichment and tissue-specific induction of circular RNA during human fetal development. Genome Biol. 2015;16:126. 015-0690-0695

27. Glazar P, Papavasileiou P, Rajewsky N. circBase: a database for circular RNAs. RNA (New York, NY). 2014:20(11):1666-70.

28. Xu T, Wu J, Han P, Zhao Z, Song X. Circular RNA expression profiles and features in human tissues: a study using RNA-seq data. BMC Genomics. 2017;18(Suppl 6):680

29. Hansen TB, Venø MT, Damgaard CK, Kjems J. Comparison of circular RNA prediction tools. Nucleic Acids Res. 2016;44(6):e58.

30. Enright AJ, John B, Gaul U, Tuschl T, Sander C, Marks DS. MicroRNA targets in drosophila. Genome Biol. 2003;5(1):R1

31. Rehmsmeier M, Steffen $P$, Höchsmann M, Giegerich R. Fast and effective prediction of microRNA/target duplexes. RNA. 2004;10(10):1507-17.

32. Kozomara A, Griffiths-Jones S. miRBase: annotating high confidence microRNAs using deep sequencing data. Nucleic Acids Res. 2014; 42(D1):D68-73.

33. Shannon P, Markiel A, Ozier O, Baliga NS, Wang JT, Ramage D, Amin N, Schwikowski B, Ideker T. Cytoscape: a software environment for integrated models of biomolecular interaction networks. Genome Res. 2003;13(11):2498-504

34. Chou CH, Chang NW, Shrestha S, Hsu SD, Lin YL, Lee WH, Yang CD, Hong HC, Wei TY, Tu SJ, et al. miRTarBase 2016: updates to the experimentally validated miRNA-target interactions database. Nucleic Acids Res. 2016; 44(D1):D239-47.

35. Agarwal V, Bell GW, Nam J-W, Bartel DP. Predicting effective microRNA target sites in mammalian mRNAs. elife. 2015;4

36. Love Ml, Huber W, Anders S. Moderated estimation of fold change and dispersion for RNA-seq data with DESeq2. Genome Biol. 2014;15(12):550

37. Farina C, Aloisi F, Meinl E. Astrocytes are active players in cerebral innate immunity. Trends Immunol. 2007;28(3):138-45.

38. Gadani SP, Cronk JC, Norris GT, Kipnis J. IL-4 in the brain: a cytokine to remember. J Immunol. 2012;189(9):4213-9. 
39. Hansen TB, Kjems J, Damgaard CK. Circular RNA and miR-7 in cancer. Cancer Res. 2013;73(18):5609-12.

40. Dropcho EJ, Chen YT, Posner JB, Old LJ. Cloning of a brain protein identified by autoantibodies from a patient with paraneoplastic cerebellar degeneration. Proc Natl Acad Sci U S A. 1987;84(13):4552-6.

41. Liang WS, Dunckley T, Beach TG, Grover A, Mastroeni D, Walker DG, Caselli RJ, Kukull WA, McKeel D, Morris JC, et al. Gene expression profiles in anatomically and functionally distinct regions of the normal aged human brain. Physiol Genomics. 2007;28(3):311-22.

42. Chen GH, Wang YJ, Qin S, Yang QG, Zhou JN, Liu RY. Age-related spatial cognitive impairment is correlated with increase of synaptotagmin 1 in dorsal hippocampus in SAMP8 mice. Neurobiol Aging. 2007;28(4):611-8.

43. Zhou X, Sun L, Bracko O, Choi JW, Jia Y, Nana AL, Brady OA, Hernandez JCC, Nishimura N, Seeley WW, et al. Impaired prosaposin lysosomal trafficking in frontotemporal lobar degeneration due to progranulin mutations. Nat Commun. 2017;8:15277.

44. Tan WL, Lim BT, Anene-Nzelu CG, Ackers-Johnson M, Dashi A, See K, Tiang Z, Lee DP, Chua W, Luu TD. A landscape of circular RNA expression in the human heart. Cardiovasc Res. 2016;113(3):298-309.

45. Zeng X, Lin W, Guo M, Zou Q. A comprehensive overview and evaluation of circular RNA detection tools. PLoS Comput Biol. 2017;13(6):e1005420.

46. Szabo L, Salzman J. Detecting circular RNAs: bioinformatic and experimental challenges. Nat Rev Genet. 2016;17(11):679-92.

47. Wang K, Singh D, Zeng Z, Coleman SJ, Huang Y, Savich GL, He X, Mieczkowski P, Grimm SA, Perou CM, et al. MapSplice: accurate mapping of RNA-seq reads for splice junction discovery. Nucleic Acids Res. 2010;38(18):e178.

48. Engström PG, Steijger T, Sipos B, Grant GR, Kahles A, Rätsch G, Goldman N, Hubbard TJ, Harrow J, Guigó R. Systematic evaluation of spliced alignment programs for RNA-seq data. Nat Methods. 2013;10(12):1185-91.

49. FastQC: A Quality Control tool for High Throughput Sequence Data [http://www.bioinformatics.babraham.ac.uk/projects/fastqc/].

50. Seqtk: a fast and lightweight tool for processing FASTA or FASTQ sequences [https://github.com//h3/seqtk].

51. Kent WJ, Sugnet CW, Furey TS, Roskin KM, Pringle TH, Zahler AM, Haussler D. The human genome browser at UCSC. Genome Res. 2002;12(6):996-1006.

52. Quinlan AR, Hall IM. BEDTools: a flexible suite of utilities for comparing genomic features. Bioinformatics (Oxford, England). 2010;26(6):841-2.

53. Dobin A, Davis CA, Schlesinger F, Drenkow J, Zaleski C, Jha S, Batut P, Chaisson M, Gingeras TR. STAR: ultrafast universal RNA-seq aligner. Bioinformatics. 2013;29(1):15-21.

54. Beach TG, Adler $C H$, Sue LI, Serrano G, Shill HA, Walker DG, Lue L, Roher AE, Dugger BN, Maarouf C. Arizona study of aging and neurodegenerative disorders and brain and body donation program. Neuropathology. 2015 35(4):354-89.

\section{Ready to submit your research? Choose BMC and benefit from:}

- fast, convenient online submission

- thorough peer review by experienced researchers in your field

- rapid publication on acceptance

- support for research data, including large and complex data types

- gold Open Access which fosters wider collaboration and increased citations - maximum visibility for your research: over $100 \mathrm{M}$ website views per year

At BMC, research is always in progress.

Learn more biomedcentral.com/submissions 